\title{
RF-Tania protocol and system architecture for location based sensor measurements
}

\author{
Soultana Ellinidou \\ Dept. of Informatics \& Telecommunica- \\ tion Engineering \\ University of Western Macedonia \\ Kozani, Greece \\ t.ellinidou@gmail.com
}

\author{
Sotirios Kontogiannis \\ Dept. of Mathematics \\ University of Ioannina \\ Ioannina, Greece \\ skontog@gmail.com
}

\author{
George Kokkonis \\ Dept. of Applied Informatics, \\ University of Macedonia \\ Thessaloniki, Greece \\ gkokkonis@uom.gr
}

\begin{abstract}
In this paper, we propose a new protocol for sensors Frequency Shift Keying data transmission named RFTania protocol framework. This protocol focuses on Energy Efficiency and it is based on an existent open source protocol stack. Tania protocol framework includes a set of three protocols: The Sensors Data Transmission Protocol (Tania-SDTP), the Ad-Hoc On Demand Protocol (Tania-AHOD) and the Adhoc Alerts Protocol (Tania-AHA), each one servicing different user functionalities.

Tania protocol is used on a specific system architecture of a crowded sensors network. Such network is composed of two different types of equipment: The measuring sensor transponders and the Ad-hoc receivers. Sensor transponders are battery operated systems with installed sensors. Ad-hoc receivers are mobile phones equipped with transponders that operate as both sensor output devices as well as sensor data retransmitters.

In the case study, a test bed system architecture for monitoring $\mathrm{CO}_{2}$ and temperature levels has been used. In this study, tests of energy endurance, RF coverage and energy performance have been performed. From the evaluation results, it is proven that RF-Tania protocol outperforms in terms of energy existing RF protocols that use periodic broadcast data transmissions.
\end{abstract}

Keywords-Wireless Sensor Networks, RF communication, Energy Efficient Operation

\section{INTRODUCTION}

$\mathrm{E}$ NERGY efficiency is a fundamental issue concerning the design of communication protocols for wireless sensor networks (WSNs). Hence, the proposed protocol focuses on that key objective.

Another important attribute of an RF sensor network is the relaxed mobility of sensors' monitoring clients. While the term mobility is a well-defined term that signifies the communication means and interoperability between clients and wireless sensors, we define the term "relaxed" as a new term. The term "relaxed" determines a wireless communication state where sensors exchange information with one another; intermediate gateways traverse sensor information to allotted servers and clients act both as sensors' data repeaters and as actual monitoring devices.
Based on these concepts a new RF protocols suite for data transmissions has been implemented. This set of protocols tries to maintain both Energy efficiency by reducing utilization of the sensors' RF transponders and MCU, while pertaining the characteristics of a relaxed mobility network architecture.

RF-Tania protocol is divided into three sub-protocols: The WSN sensor protocol for the broadcasting of sensor data, the WSN client protocol, for the reception and acknowledgement of sensor protocol messages and the WSN alerts protocol for the transmission of sensor critical information. The major function of RF-Tania is to save energy by putting the sensor transmitter into sleep-mode, when the sensors measurements remain constant between two or more consecutive periods. As far as the transmitted measurements are concerned, the RF-Tania protocol forces the receivers to handle frame re-transmission, whenever the sensor measurement remains at a constant level (Sensors Data Transmission Protocol, Tania-SDTP).

The mobile user requests a sensor's measurement through a mobile phone client application. If the sensor is in sleep mode, the nearby clients will inform the client that issued the request about the last sensor measurement (Ad-Hoc On Demand Protocol, Tania-AHOD).

Ultimately, Tania-RF protocol is capable of informing all WSN nodes whenever sensor measurement or measurements correlation function is higher or equal to a threshold value. In such alert cases the transmitter will immediately send an alert-message to all connected receivers in the network (Ad-Hoc Alerts Protocol, Tania-AHA).

RF-Tania protocol is comprised of 3-layer according to the OSI model. The physical layer includes a FrequencyShift Keying (FSK) data transmission. The Data link layer is consisted of a MAC layer of node broadcast frames called Jeelab frames [1]. The network layer includes the RF-Tania protocols.

This paper structure is organized as follows: In section II we discuss the related work, in section III we present the proposed protocols suite, in section IV we provide the implementation of protocol and in section $\mathrm{V}$ we conclude to 
our contribution and present a future system and protocol modifications.

\section{RELATED WORK}

There is a wide number of WSN energy efficient protocols seen in the literature. A comprehensive classification and survey on this topic presented in [2]. These protocols work on the assumption that energy is limited and exhaustible. Consequently, the effort is primarily shifted towards prolonging the network lifetime. However, with energy harvesting capability [28], there is a need of fresh perspective on protocols and network design. Specifically, in the scenario of RF energy transfer, the protocol proposed in [6], and its subsequent analytical model in [7], adopts a duty-cycle based on the proportion of harvested energy.

The authors in [3] proposed a Medium Access Control (MAC) protocol, called RF-MAC, which ensures optimal energy delivery to the requesting node. In RF-MAC, a node broadcasts its request for energy harvesting (RFE) frames containing its ID, and then waits to hear for the Energy feeding Transmitters (ETs) in the neighborhood.

In [4], the authors proposed a network architecture which consists of two types of RF sensors. One class of sensors harvests RF energy on the DTV band $(614 \mathrm{MHz})$, while another uses the $915 \mathrm{MHz}$ ISM band. The energy transfer stage begins when the ET sends out the Request to Charge (RTC) packet, at $915 \mathrm{MHz}$ (operating frequency of Mica2 mote), offering to transfer wireless energy to the sensors in the network. Consequently, both types of devices can hear the RTC packet sent by the ET. The sensors that received the RTC then acknowledge this packet by sending back a response, called energy pulses. Once the ET receives the energy pulses from the responding sensors, it estimates the average power that sensors will receive during the charging process.

In [7] the authors implemented the WirelessHart (Highway Addressable Remote Transducer), which is a digital protocol for two-way communication between a host application and smart field instruments, providing access to diagnostics, configuration and process data. It specified a physical layer which used FSK to superimpose digital communication signals at a low level of 4-20mA. It supports two types of networks: Point to point and multi-hop network. WirelessHart is an RF protocol used by industrial vendors such as ABB and OMRON (PLC). It prevents message collisions using a TDMA use of the radio channel on predefined time slots instead of sensing the medium to transmit (CSMA/CA-CSMA/CD). However, its adoption to industrial application limits the feasibility for use in commercial and residential application due to its increased deployment cost [10].

Synkro RF network specification was developed by Freescale [11]. Synkro is mainly implemented for ad-hoc and low cost sensors. It offers a maximum transmission rate of
$250 \mathrm{kbit} / \mathrm{s}$, three independent channels in the $2.4 \mathrm{GHz}$ band and two network node types: a controller and controlled nodes. The main weakness over its PAN alternatives is the maximum number of 32 controlled nodes per controller (similar to Jeelab implementation). Moreover, the SMAC protocol [6] is used for developing proprietary RF transceiver applications using a Freescale 802.15.4 transceiver. It supports point to point communications by having a verylow power, proprietary, bi-directional RF communication link between nodes. Freescale is porting its protocol and network specification into home appliances such as DVDs and TVs offering a two-way communication alternative, in order to replace existing infrared technologies.

Bluetooth Low Energy is a $2 \mathrm{MHz}$ BW protocol at 2.4 $\mathrm{GHz}$ and uses TDMA as a medium access mechanism (IEEE 802.15). It uses a low to medium power transmission of -20 up to $10 \mathrm{dBm}$ in comparison with Bluetooth Class $1 / 2 / 3$ accordingly [12, 13, 16]. ZigBee [15] is a Bluetooth alternative protocol created for industrial applications. It uses a variety of IEEE 802.15.4 [14] standard and the same frequency band with Bluetooth and BLE. ZigBee is more of a mesh wireless protocol rather than a $\mathrm{P} 2 \mathrm{P}$ protocol such as BLE [16].

The RF4CE [5, 8] is a convenient, low-cost, low-power wireless transmission protocol used both by the ZigBee company and the RF4CE association. It is built from the standard specs of the networking layer and the application layer is implemented by the IEEE 802.15.4. It differs from ZigBee, as it does not have a complicated Internet routing protocol or multiple transmission communication mechanisms. It operates in the $2.4 \mathrm{GHz}$ frequency band utilizing three RF channels with a total $\mathrm{BW}$ of $2 \mathrm{MHz}$ [8].

WiMedia Ultra-WideBand (UWB) radio platform uses frequencies from $3.1 \mathrm{GHz}-4.85 \mathrm{GHz}$, or at $6.2 \mathrm{GHz}-$ 9.7GHz [18]. WiMedia frames may be sent in either unicast or broadcast fashion. Unicast frames are directed to their destination based on a 16-bit device address; certain addresses are also reserved for broadcast groups. WiMedia Ultra Wide Band (UWB) is a short range high throughput technology using Tx Power of (12-20dBm) covering a maximum range of $10-20 \mathrm{~m}$ with speeds up to $500 \mathrm{Mbit} / \mathrm{s}$. It is commonly used for multimedia applications.

Aside from the WiMedia implementation, an extension of IEEE 802.15.4, IEEE 802.15.4a-2007 uses the unlicensed Gigahertz bands. The standard provides two physical protocols the UWB PHY and Crisp Spread Spectrum - CSS PHY. UWB provides high data rate channels in three distinct unlicensed $\mathrm{GHz}$ bands, providing speeds of $110 \mathrm{~Kb} / \mathrm{s}$ up to $26 \mathrm{Mb} / \mathrm{s}$ and maximum range of $100 \mathrm{~m}$, with the optional feature of precision ranging(used for indoor positioning) [19]. CSS PHY on the other hand, is a low power consumption, low range and low data rate alternative used at the $2.4 \mathrm{GHz}$ band for sensor data transmissions up to $1 \mathrm{Mb} / \mathrm{s}$ [19, 20, 22]. 


\section{PROTOCOLS SUITE FOR SENSORS DATA TRANSMIS- SION}

The main purpose of the RF-Tania protocols suite is to save energy by reducing the utilization of the sensors' RF transponders and MCU, while maintaining relaxed mobility network architecture.

Our protocols suite has been implemented on the RF12 driver, used for the RFM12B JeeNode module [1]. The RF12 driver operates as follows:

- Nodes can only communicate with each other if they are in the same "net group" (1-250)

- Nodes in the same net-group have a unique $\operatorname{ID}(0 . .31)$

- Frames (Jeelab frames) are 0-66 bytes long

- Frames utilize extra 9 bytes of overhead, including the preamble

- Data is sent at a maximum rate of $45-50 \mathrm{Kbit} / \mathrm{s}$

The RF-Tania protocol stack consists of 3-layers, according to the OSI model: Physical layer, Data link layer, Network layer. Each one of these layers is explained below.

\section{A. Network Architecture}

Network architecture consists of two entities, the transmitter and the receiver, as we can see in Fig 1. Specifically, the transmitter or sensor entity contains RF-transponder and microcontroller equipped with sensors. The receiver or mobile client entity contains mobile phones equipped with RFtransponders.

The transmitter, called sensor entity, is placed on a stable position transmitting the sensors measurements to the mobile receivers. The mobile receivers, called client receivers, are moving around. The sensor entity transmits data every period $T_{p}$ to all mobile receivers, located in the coverage area.
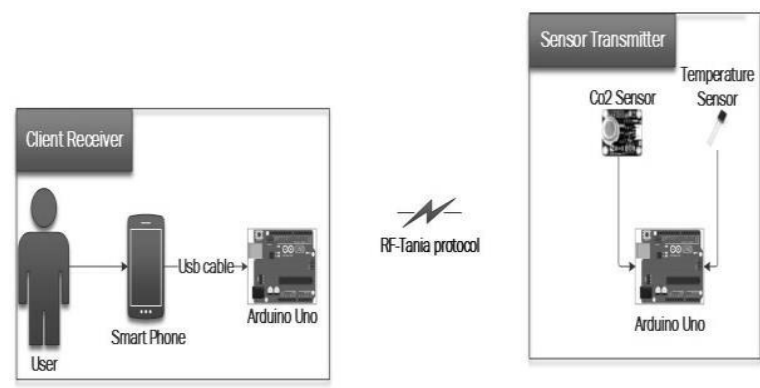

Fig.1: WSN receiver and sensor entities

In the WSN network we use a series of RFM12B transponders. Technical characteristics of RFM12B chip are outlined at [23] and [24] for the long-range transceivers.

\section{B. Physical Layer}

The RFM12B initialization includes the communication transponder used for our WSN network. The physical layer includes the following configuration fields: Configuration Settings, Power Management, Frequency Settings, Data Rate and the Receiver Control.
As far as the configurations settings are concerned we used the band of $433 \mathrm{MHz}$, in order to minimize interference with other transmissions in the area (GSM frequencies, WiFi, Bluetooth and GPRS data transmissions). Furthermore, we can activate the RX Register and TX FIFO Buffer. These chip configuration settings parameters are set from the Arduino microcontroller (sender or receiver entity) via the SPI (Serial Peripheral Interface), sending 16bit commands to the RFM12B chip.

The parameters control the power to the RFM12 submodules and allow the selection of circuits, until the RFM12 is turned on or turned off accordingly. So by disabling these circuits, when it is not required, we can control the amount power consumption of the device.

The RFM12B chip has the ability of frequency hoppingshifting to nearby frequencies. The default carrier frequency shifting is $90 \mathrm{KHz}$. If there is an interference on one frequency, another frequency can be selected manually and interference problems can be avoided.

The RF12B incorporates a fully integrated Power Amplifier (PA) with antenna tuning and a Low Noise Amplifier (LNA) with switchable gain. The Power Amplifier (PA) has an open-collector differential output and can directly drive a loop antenna with a programmable output power level of maximum of $0 \mathrm{dBm}$ transmission power.

An automatic antenna tuning circuit is built in to avoid costly trimming procedures and the so-called "hand effect". The Low Noise Amplifier (LNA) has approximately $250 \mathrm{Ohm}$ input impedance, which functions well with the proposed $\lambda / 4$ monopole antennas (of $17.3 \mathrm{~cm}$ length). If the RF input of the chip is connected to $50 \mathrm{Ohm}$ devices, an external matching circuit will be required to provide the correct matching and to minimize the noise figure of the receiver. The LNA gain can be selected in four steps (between 0 and $-20 \mathrm{dBm}$ ) according to RF signal strength required.

The Data Rate command sets the bitrate of the transmitted data or the expected bitrate of the received data. The actual bit rate in transmit mode and the expected bit rate of the received data stream in receive mode are determined by the 7-bit parameter R (bits r6 to r0) and bit cs. The highest data rate is set at $57.4 \mathrm{Kbit} / \mathrm{s}$. This gives $17 \mathrm{usec}$ per bit transmission and 139 usec per byte $(7192 \mathrm{Kbyte} / \mathrm{s}$ or $57,553 \mathrm{Kbit} / \mathrm{s})$.

The Receiver Control command contains a series of various bits, according to [25]:

Bit 10 (P20): sets the function of INT/VDI pin on the RFM12 module. It configures the module as input (Interrupt from MCU) or output (VDI Valid Data Indicator).

Bits 9-8 (d1 to d0): VDI (valid data indicator) signal response time setting

Bits 7-5 (i2 to i0): Receiver baseband bandwidth (BW). The receiver bandwidth is selectable by programming the bandwidth (BW) of the baseband filters. The bandwidth settings are linked to both data rate, and $\mathrm{Tx}$ modulation 
commands. When data rate is fast a higher receiver bandwidth is required. The default baseband BW of the RF12B is set to $134 \mathrm{KHz}$. The highest receiver $\mathrm{BW}$ is set to 450 $\mathrm{KHz}$ [24].

Bits 4-3 (g1 to g0): LNA (Low Noise Amplifier) gain $(\mathrm{dBm})$. Typically, OdBm is the LNA output of the RFM12B chip. Consequently, the maximum transmission power of the transponder is $0 \mathrm{dBm}$.

Bits 2-0 (r2 to r0): bits that set the RSSI detector threshold. The RSSI threshold is based on the $433 \mathrm{MHz}$ beacon carrier and expresses how strong or weak the transmitter beacon signal is (as spotted at the receiver end). If it is below a certain threshold (set by bits) the receiver will ignore the incoming transmitted frame.

\section{Data Link Layer}

The Data link layer includes the MAC layer, which is responsible for controlling how and when network devices gain access to medium and permission to transmit data. The nodes broadcast frames, called Jeelab frames constructed in this layer.

The Carrier Sense Multiple Access with Collision Avoidance (CSMA/CA) is a network multiple access method in which carrier sensing is performed. The nodes attempt to avoid collisions by transmitting data only when the channel is sensed to be idle. Moreover, a rule of $1 \%$ channel utilization applies at the data link layer. This means that each transmitting node in our network should send up to $1 \%$ of the time, on average. The $1 \%$ rule is a very simple collision avoidance mechanism. There is also a random back off collision avoidance mechanism included in Jeelabs library whenever a collision occurs in cases of frames retransmission. Both of these mechanisms are implemented on the rf12_easy transmit Jeelab's library function [1].

The original frame header, implemented by Jeelabs [1] is shown in Fig. 2 (Preamble, SYN, Head and CRC). The proposed protocol suite header field is encapsulated in a Jeelab frame, shown in Fig. 3 (Packet_ID, Node_Id Measurements and Send_time).

The Preamble and SYN fields are used for data transmission synchronization and group selection (Each group can contain up to 32 nodes with unique node ids). The Head field includes Jeelab protocol options and source or destination node id. The frame Payload is 0-56bytes. The CRC (Cyclic Redundancy Check) is the error-detecting code field.

In order to transmit frames, RF-Tania protocol uses the Head field of the original Jeelabs frame. There are three bits: $\mathrm{C}=\mathrm{CTL}, \mathrm{D}=\mathrm{DST}, \mathrm{A}=\mathrm{ACK}$ and a 5-bit node ID. (Fig. 2 - Head field). Node id values can be 0-31. The A bit (ACK) indicates whether this frame wants to get an ACK back. The $\mathrm{C}$ bit needs to be zero in this case. The $\mathrm{D}$ bit (DST) indicates whether the node ID specifies the destination node or the source node. For frames sent to a specific node, DST = 1 (The destination node id is included in the Head field).

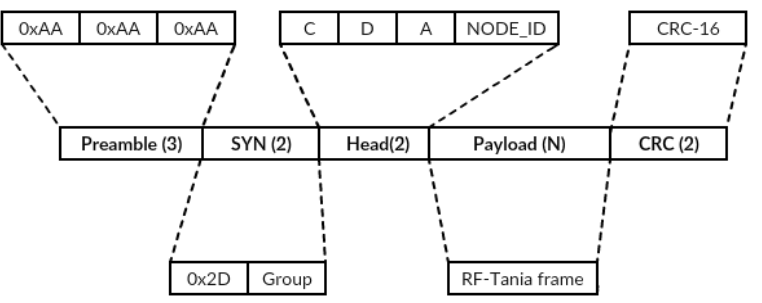

Fig 2: The Jeelab frame header [1]

For broadcasts, DST $=0$, in which case the node ID field refers to the originating node. The $\mathrm{C}$ bit (CTL) is used to signify ACK request and should be combined with the A bit set to zero. The node receiving an ACK can check the originator of the ACK reply.

As for the Payload field of the Jeelab frame, we propose the format shown at Fig. 3. The first field of RF-Tania payload contains an auto incremented number of frame ID. The second field carries the ID of the sending node and it is 5 bits (3 bits left for future use possibly QoS or Collision experience provisions). The Measurements field includes 4 bytes sensor measurements and the Send_time field includes a 4 bytes timestamp of the frame. In case of sensor entity measurement, Send_time is represented by the MCU millis time.

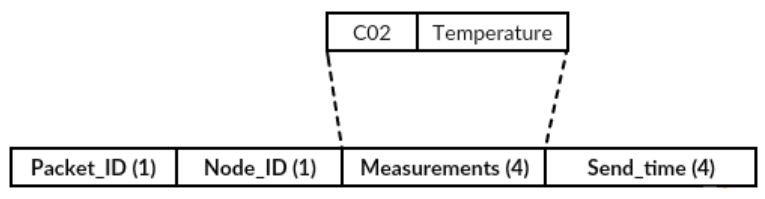

Fig. 3: RF-Tania frame

RF-Tania frame can include simultaneously 24 measurements of 2 bytes each one ( 48 bytes overall) or 12 measurements of 4 bytes each one. In our case study we use 2 measurements of 4 bytes from a $\mathrm{CO}_{2}$ and Temp sensor accordingly.

\section{Network Layer}

The network layer includes the RF-Tania protocol, which is categorized into 3 sub-protocols:

Tania-SDTP: According to SDTP protocol the receiver entity periodically collects sensor measurements from the sensor entities (Fig. 4). Each sensor entity collects measurements from a number of sensors connected to the entity. The sensor entity device has two periods: the sensor device data collection period $\left(\mathrm{T}_{\mathrm{sc}} \approx 2 \mathrm{~s}\right)$ and the sensor device network data transmission period $T_{p}$ (initially $T_{p}=30 s$ ). For a period $\mathrm{T}_{\mathrm{p}}$, all sensors measurements retrieved from a sensor entity, are stored in the sensor entity SRAM. At the end of each period $T_{p}$, the sensor entity calculates the mean sensor values using equation (1) and checks whether equation (2) is satisfied:

$$
x_{p}=\frac{1}{n} \sum_{i=1}^{n} x_{C i}
$$




$$
\left(\left|\frac{x c_{i}-x c_{i-1}}{d t}\right|\right)_{T c} \geq 4 \min \left(\left|\frac{d x p}{d t}\right|\right)_{T p}
$$

In the above functions, $x c_{i}$ is one sensor measurement at time $\mathrm{T}_{\mathrm{sc}}(\mathrm{i}=1$...n sensor measurements collected in a time interval $\left.\mathrm{T}_{\mathrm{p}}\right), \mathrm{x}_{\mathrm{pi}}\left(\mathrm{x}_{\mathrm{p}}\right)$ is the mean calculated sensor measurement ready for transmission, $\mathrm{xp}_{\mathrm{i}-1}$ is the previously transmitted sensor measurement. If equation (2) is satisfied, the current sensor mean measurement will be considered of high variance and an alert will be set by the sensor entity using Tania AHA protocol. A sensor entity alert forces the sensor entity to perform a multiplicative decrease of its transmission period $\left(\mathrm{T}_{\mathrm{p}}=\mathrm{T}_{\mathrm{p}} / 2, \mathrm{~T}_{\mathrm{p}}>2 \mathrm{~s}\right)$. Transmission period value multiplicative decrease is performed in each transmission interval until equation (2) is satisfied. If equation (2) does not apply, the sensor entity will perform an increase of the transmission period $T_{p}$ using the following equation: $T_{p}=T_{p}+1 \times f_{p}(s e c)$, where $f_{p}$ is the sensor data transmission frequency coefficient.

In a previous data transmission interval $\mathrm{T}_{\mathrm{p}}$, a sensor entity collected n number of measurements (M) from a $\mathrm{CO}_{2}$ sensor in period $\mathrm{T}_{\mathrm{sc}}<<\mathrm{T}_{\mathrm{p}}$. For the $\mathrm{T}_{\mathrm{p}}$ period interval the maximum measured $\mathrm{CO}_{2}$ sensor value is $\mathrm{M}_{\max }$ and the minimum measured value is $\mathbf{M}_{\min }$ (Equation (3)). For each transmission period, a frequency coefficient parameter $f_{p}$ is calculated based on equations (3) and (4) and used for the determination of the next period $\mathrm{T}_{\mathrm{p}}$ value.

$$
\begin{gathered}
c p=\frac{M_{\max }^{T p}-M_{\min }^{T p}}{\frac{1}{n} \sum_{i=1}^{n-1}\left[M_{T s c}^{i}-M_{T s c}^{i-1}\right]} \\
f_{p}=\frac{1}{c p}
\end{gathered}
$$

$\mathrm{M}_{\mathrm{TSC}}$ values are the sensor measurements per time intervals called ticks $\left(\mathrm{T}_{\mathrm{sc}}\right)$. The denominator of equation (3) expresses the average $M_{T S C}$ value for a period $T_{p}$. If $f_{p}<1, f_{p}$ will be set to 1 . Coefficient $\left(c_{p}\right)$ is called energy efficient parameter. When its value decreased, the sensor will become less sensitive to measurement variations or sensor variations are minimum. Moreover, when the energy efficient parameter value decreased, the sensor values variations will be significant and at the same time the sensor entity will spend more energy for data transmission.

If equation (2) is satisfied, the sensor entity will send alerts to the receiver entities using Tania AHA alerts protocol. As soon as a frame is sent to the receiver, it should send back an ACK frame to the sensor entity (see Fig. 6).

Tania-AHOD: This is the on demand measurements request protocol. Specifically the client-receiver, which is connected to the users' mobile-phone, requests measurements from a sensor entity. In that case, either the sensor entity (if it is in listen mode-Fig. 4, Tania on demand request listen period) might respond to the request or another nearby receiver entity might respond. In case of reception of multiple responses by the receiver entity, the receiver will choose the sensor entity responses first. If nobody response, the receiver entity will response with the most current timestamp field in its Tania header frame.

Once the receiver makes a decision about the best frame to keep, then it will forward it to the mobile phone application layer. The requested measurement will appear on user mobile display (see Fig. 5).

Tania-AHA: This protocol is responsible for informing all receiver nodes for critical measurement values. Sensor alerts occur whenever sensor measurements satisfy the equation (2) or the sensor measurements are higher or equal to a threshold value.

For example, in a sensor network that monitors environmental $\mathrm{CO}_{2}$ values, the sensor entity collects $\mathrm{CO}_{2}$ sensor data. Furthermore, it is checking once for every period $T_{p}$ whether equation (2) is satisfied or the mean sensor value for that period is above threshold value of 1000ppm for $\mathrm{CO}_{2}$ (see Fig.6). If equation (2) is satisfied or mean sensor value is above threshold value then the sensor entity will immediately send an alert-message to all connected receiver entities in the WSN network using unicast transmissions (Alert frames will be sent to all sensor nodes that acknowledged last periodic sensor entity transmission using Tania-SDTP protocol). As soon as the alert-messages are sent, the receiver entities will open a serial connection with mobile phone, in order to inform the user (Fig. 6).

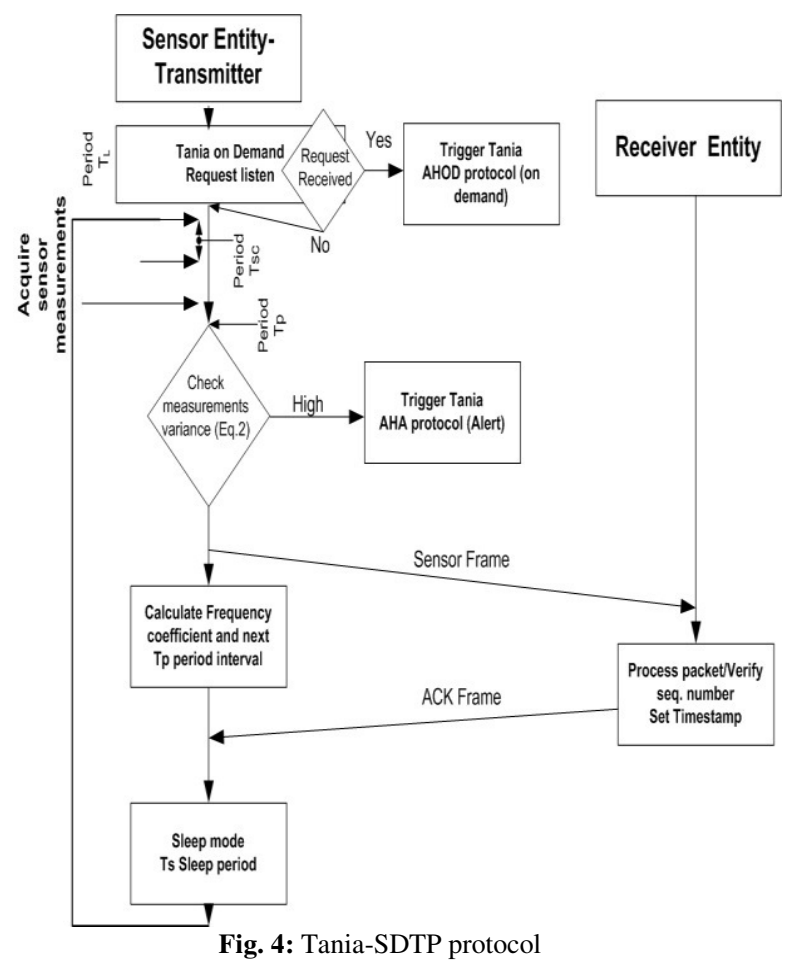

The Tania AHA alerts transmission phase is persistent and energy consuming. If a receiver entity does not acknowledge the reception of the alert frame, the alert message transmission will be broadcasted and the alert broadcast will be repeated every $1 \mathrm{sec}$ for a period $\mathrm{T}_{\mathrm{p}}$ equal to the period of $30 \mathrm{sec}$ ( 30 alerts back to back). This step is per- 
formed until at least one receiver entity acknowledges the alert reception to the sensor entity.

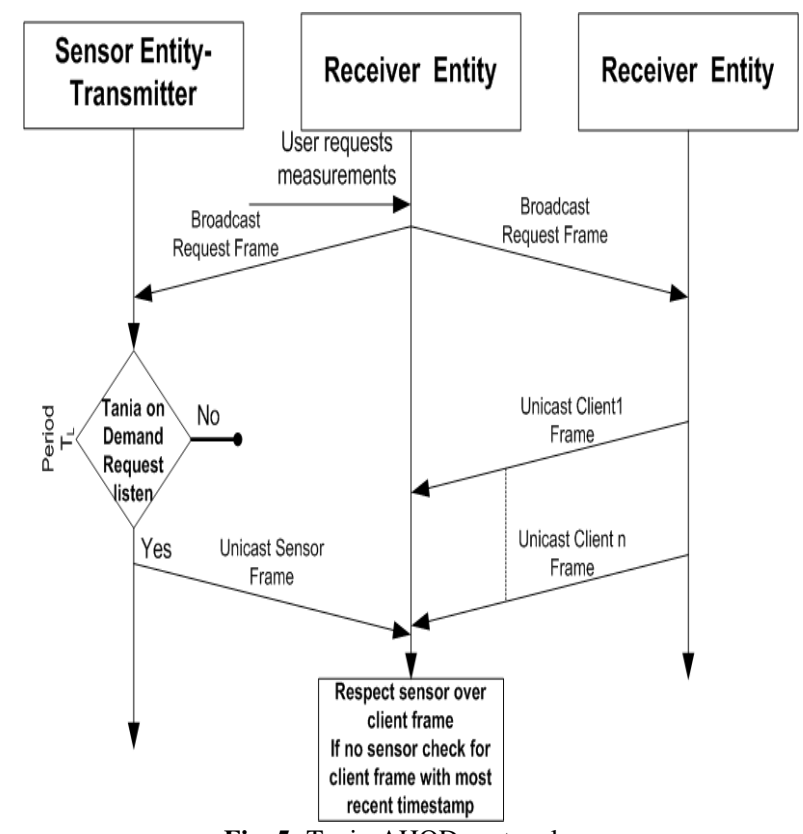

Fig. 5: Tania-AHOD protocol

The sensor entity timeout value for all unicast acknowledgements reception is set to $2 \mathrm{sec}(\mathrm{RTO}=2 \mathrm{~s})$. If $\mathrm{RTO}$ is reached, a frame alert retransmission for non-acknowledged nodes will be performed at least three times for each node. As soon as the alert frame transmission is completed, a multiplicative decrease in transmission period will be performed.

\section{CASE STUDY AND PERFORMANCE MEASURE- MENTS}

The case study includes one sensor entity (see Fig. 1 MCU transmitter with temperature and $\mathrm{CO}_{2}$ sensors). The sensor entity equipment uses a $3.3 \mathrm{~V}$ Arduino pro mini microcontroller connected to an MG-811 C02 analog sensor and a DS18B20 digital temperature one-wire sensor. On the Arduino SPI pins the RF12B transponder is connected in order to transmit periodically measurements to the WSN network. The WSN network consists of two receiver entities nodes. These nodes include an Arduino UNO 5V microcontroller with an SPI RFM12B transceiver. The Arduino is back to back connected via OTG cable to Android mobile phones where the measurements monitoring application resides. The application measurements real time is set by the mobile phones as soon a measurement is received.

The Arduino board used at sensor entities operates at 3.3 $\mathrm{V} \mathrm{DC}$, since there is a $\mathrm{CO}_{2}$ sensor requirement to operate at $5 \mathrm{~V} \mathrm{DC}$ the sensor is powered directly from the battery unit. The typical power consumption of an Arduino UNO $5 \mathrm{~V}$ board in idle mode is $282-300 \mathrm{~mW}$. However, the same board Arduino pro mini, operated at $3.3 \mathrm{~V}$ provides power requirements at idle mode of $27-35 \mathrm{~mW}$, including the volt- age regulator $5 \mathrm{~V}-3.3 \mathrm{~V}$ chip power consumption (10 times less than an Arduino5V UNO board used at primer experimentation).

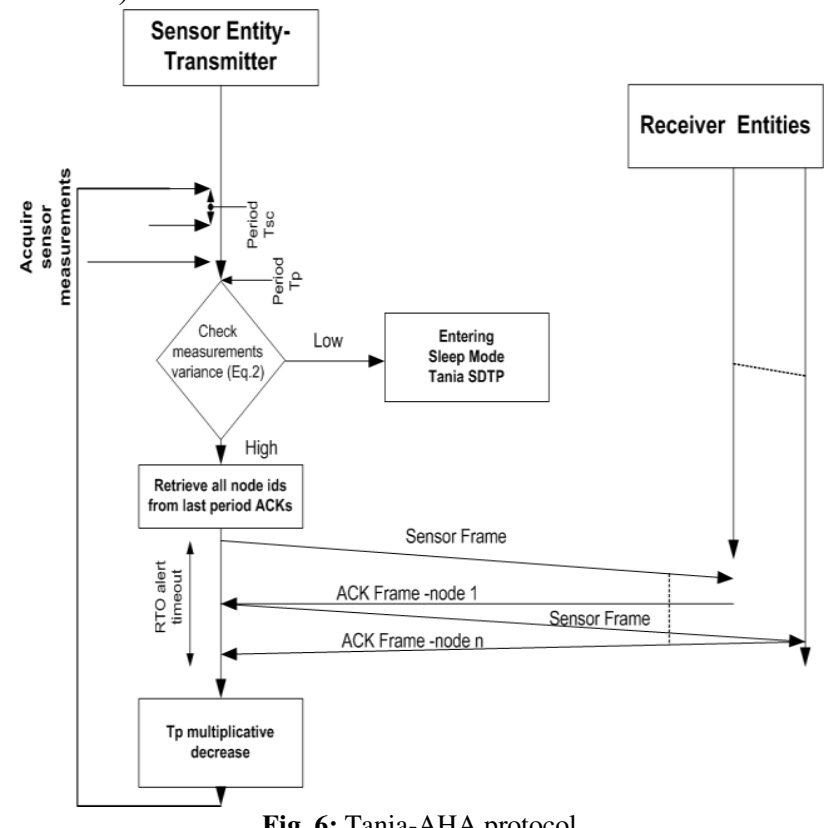

We are using an MG-811 $\mathrm{CO}_{2}$ sensor [25], which includes a signal conditioning circuit along with a LNA amplifier. Its power consumption is rated from $15-18 \mathrm{~mA}$, thus giving a power consumption outcome for $5 \mathrm{~V}$ sensor required voltage of $\max 90 \mathrm{~mW}$. This is still 3 to 4 times less than Arduino UNO idle power consumption. On the other hand, both digital thermometer DS18B20 and RF12B transponder operate at $3.3 \mathrm{~V}$, with transmission RF12B power consumption of $76 \mathrm{~mW}$ and thermometer consumption of $6 \mathrm{~mW}$.Moreover, more power-enhanced improvements have been performed by providing a low power consumption algorithm for the sensor entity. The algorithm's description follows.

Arduino board has six different sleep mode states: idle, ADC noise reduction, power-down, power-save, standby and extended standby [26]. Power save and power down modes power off all Arduino peripheral chips such as SPI, TWI and ADC, contrary to idle mode that still consumes power. Both power save and power down can be triggered by external Interrupts (INT0 and INT1). However, power save mode still feeds power to Arduino timers (0/1/2), while power down mode disables their functionality completely. This of course leads us to Arduino timing problems (millis function connected to timer0) [26].

Arduino includes a watchdog timer (WDT). This timer has a separate on chip RC clock operating at $128 \mathrm{KHz}$ and has 9 prescaler modes of operation starting from $30 \mathrm{~ms}$ up to 8 s. From these modes authors selected the following sleep interval modes to be configured at their algorithm's initialization step: $1 \mathrm{~s}, 2 \mathrm{~s}, 4 \mathrm{~s}$ and $8 \mathrm{~s}$. WDT timer is functional on all Arduino power modes (including power down). 
The Brown out detector (BOD) [26] is another functionality of the Arduino board that consumes power and is not required for the sensor entity. The BOD detector checks for voltage anomalies or dips in the voltage of the board and resets the Arduino chip by calling the watchdog timer. This BOD functionality is set by the Arduino fuse bits (extended fuse bit $0 x 05$ to $0 x 07$ ) and is programmatically pushed to the sensors using in circuit programmers. This BOD functionality reduces the board energy consumption from 0.1 to $0.3 \mathrm{~mW}$ of continuous power. Moreover, removing the Arduino power led also contributes to less power consumption of $10-12 \mathrm{~mW}$.

We propose the following low power reduction steps for our sensor entity measurements:

Step 1a-Setup step: Arduino disables the watchdog timer and initializes timer0 and RFM12B transmission parameters. The $\mathrm{CO}_{2}$ board is powered directly from $6 \mathrm{~V}$ battery pack as well as the Arduino Pro mini. RFM12B is powered from Arduino Pro mini Digital pin 5 set to $\mathrm{HIGH}$ (3.3V), DS18B20 is powered from Digital pin 6 set to HIGH (3.3V). $\mathrm{T}_{\mathrm{sc}}$ period counter is set to zero, millis() value is at $5000 \mathrm{~ms}(5 \mathrm{~s})$.

Step 1b-Sensor main loop: This is the initiation of the MCU sensors measurement process of sensor probing and it is repeated every $\mathrm{T}_{\mathrm{sc}}$ time interval set at $2 \mathrm{sec}$.

Step 2a - Check for Ad-hoc requests: Arduino opens the RFM12B transponder for 100ms and checks for any Ad-hoc on demand requests. If a request is sensed it transmits data to the request node.

Step $2 \mathbf{b}-\mathbf{T}_{\mathbf{p}}$ period reached: Arduino checks whether the periodic counter value of sensor measurements initially set at $\mathrm{T}_{\mathrm{p}}=30 \mathrm{sec}$ has elapsed. If it has elapsed, it will initialize the SPI interface and transmits sensor data via the RFM12B transponder. Afterwards, it opens an I2C communication with the EEPROM chip, an Atmel AT24C128 I2C EEPROM chip [27], reading past period measurements and performs calculation (period increase or decrease) of the next period interval based on the algorithm described at section III.D.

Step 2c - Sensors probing Loop: If $\mathrm{T}_{\mathrm{p}}<30$ or $\mathrm{T}_{\text {pcalculated, }}$ Arduino enters a probing Loop, sets its ADC frequency to $125 \mathrm{KHz}$ and performs sequentially 96 measurements of $\mathrm{CO}_{2}$ and 96 measurements of temperature (around $2 \mathrm{~ms}$ of time). The time completion and averaging result is produced and saved to an external EEPROM memory I2C communications chip [27].

Step 3 - Sensor MCU Sleep mode: Watchdog timer is set for time of 1s with interrupt 1 enabled. The Arduino goes to power down mode and the digital pins 5,6 are set to low. The WDT timer is set as the main millis timer (128 times slower counting - for time period of 1s WDT increases millis value by a value of 8 instead of 1000).

Step 4 - Sensor MCU wake up: Interrupt service routine of Interrupt 1 is entered disabling the watchdog tim- er and setting the Arduino chip out of the power down mode.

Step 5 - MCU clock calibration: End of Microcontroller loop, set millis counter to millis()+1000-8. $\mathrm{T}_{\mathrm{sc}}$ period counter calculation equal to (millis()-5000)/1000 and loop re-start from step $2 \mathrm{a}$.

TABLE 1:

PROPOSED SENSOR ENTITY ARCHITECTURE COMPONENTS AND POWER CONSUMPTION

\begin{tabular}{|c|c|}
\hline Equipment & $\begin{array}{c}\text { Power Consumption- } \\
\text { idle mode }\end{array}$ \\
\hline $\begin{array}{c}\text { Arduino UNO 5V } \\
\text { Arduino Pro mini 3.3V -idle } \\
\text { state }\end{array}$ & $33 \mathrm{~mW}$ \\
\hline MG-811 sensor 5V & $90 \mathrm{~mW}$ \\
\hline DS18B20 & $6 \mathrm{~mW}$ \\
\hline RFM12B transmission & $76 \mathrm{~mW}$ \\
\hline $\begin{array}{c}\text { RFM12B data reception, ad- } \\
\text { hoc sensing }\end{array}$ & $39.6 \mathrm{~mW}$ \\
\hline \begin{tabular}{c} 
AT24C128 EEPROM \\
\hline
\end{tabular} & $17 \mathrm{~mW}(100,000$ writes $)$ \\
\hline
\end{tabular}

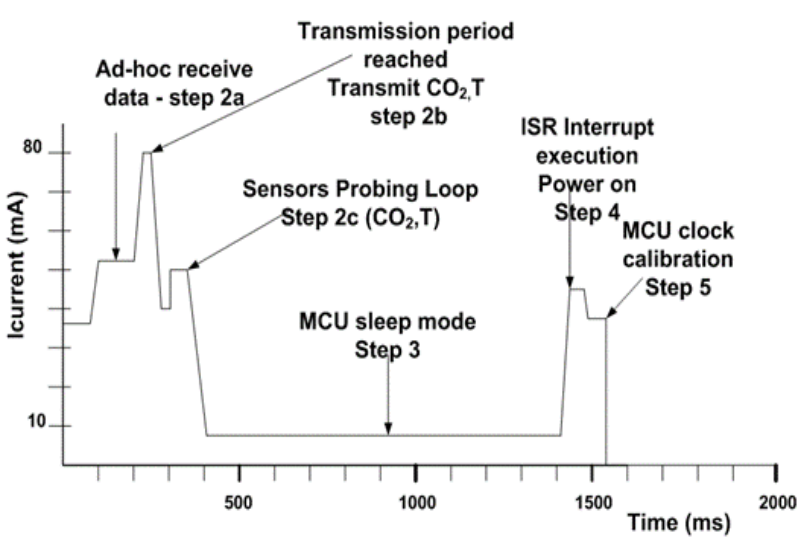

Fig. 7: Sensor entity energy footprint for a $\mathrm{Tp}=2 \mathrm{sec}$.

TABLE 2:

WSN COVERAGE AREA FRAME LOSS AND MAXIMUM CHANNEL CAPACITY

\begin{tabular}{|c|c|c|c|}
\hline $\begin{array}{c}\text { Coverage } \\
\begin{array}{c}\text { Distance } \\
\text { (meters) }\end{array}\end{array}$ & $\begin{array}{l}\text { Frame } \\
\text { loss }\end{array}$ & $\begin{array}{c}\text { Mean delay } \\
\text { (sec) }\end{array}$ & $\begin{array}{c}\text { Throughput } \\
\text { (bits/sec) }\end{array}$ \\
\hline 10 & $0 \%$ & 0.08 & 2373.5 \\
\hline 20 & $0 \%$ & 0.1 & 2362.3 \\
\hline 30 & $3 \%$ & 0,15 & 2286.2 \\
\hline 40 & $7 \%$ & 0,19 & 2187,6 \\
\hline 50 & $15 \%$ & 0,22 & 1996,5 \\
\hline
\end{tabular}

From the sensor entity and WSN experimentation the maximum energy footprint for a minimum sensor entity period of $T_{p}=2 s$ is presented at Figure 7. In addition, the WSN sensor pin powered RFM12B transponder-receiver RFM12B transponder maximum coverage distance and 
maximum channel throughput capacity are shown at Table 1 .

The WSN sensor pin powered RFM12B transponder-receiver RFM12B transponder maximum coverage distance and maximum channel throughput capacity are shown at Table 2 .

\section{CONCLUSIONS}

This paper presents a new WSN architecture comprised of sensor entities and mobile receiver entities, for the transmission of sensory data to mobile roaming clients with medium to small transmission ranges coverage. Our approach is based on existing cheap and open source hardware and software solutions. On the proposed WSN architecture, a set of three network layer protocols is presented, called RF-Tania protocol framework, for the transmission of relaxed sensory data, ad-hoc on demand transmission of sensory data and alert transmissions in cases of sensors' threshold events.

It is set as a future work, a further detailed experimentation of proposed architecture and protocols, as well as comparison results of the sensor entity energy consumption and coverage with similar transponder devices energy consumption and coverage results.

\section{REFERENCES}

[1] Jeelabs, RF12B MAC protocol, http://Jeelabs.org, 2013.

[2] Z. A. Eu, H-P. Tan, and W. K. G. Seah, "Design and performance analysis of MAC schemes for wireless sensor networks powered by ambient energy harvesting". Ad Hoc Networks, vol. 9, no. 3, ISSN 1570-8705, pp. 300-323, 2011.

[3] M. Y. Naderi, P. Nintanavongsa,K. R. Chowdhury, "RF-MAC: A Medium Access Control Protocol for Re-Chargeable Sensor Networks Powered by Wireless Energy Harvesting".IEEE Transaction on Wireless Communications, vol. 3, issue 7, ISSN 1536-1276, pp. 3926 -3937, July 2014.

[4] P. Nintanavongsa, M.Y. Naderi, and K. R. Chowdhury, "A Dual-band Wireless Energy Transfer Protocol for Heterogeneous Sensor Networks Powered by RF Energy Harvesting".International Conference on Computer Science and Engineering Conference (ICSEC),IEEE,pp.400-405, Sept. 2013.

[5] Dong-feng, Su, C. Xiang-jian, L. Di, X. Zhi-jun and C. Zhi-feng, "Research of New Wireless Sensor Network Protocol: ZigBee RF4CE."International Conference on Electrical and Control Engineering (ICECE), IEEE, pp. 2921-2924, 2010.

[6] Song, Wen-Miao, Yan-Ming Liu, and S-E. Zhang. "Research on SMAC protocol for WSN." International Conference on Wireless Communications, Networking and Mobile Computing, IEEE, pp. 1-4, 2008.

[7] Song, Jianping, S. Han, A. Mok, D. Chen, M. Lucas M. Nixon and W. Pratt, "WirelessHART: Applying wireless technology in real-time industrial process control." Real-Time and Embedded Technology and Applications Symposium, IEEE, pp. 377-386, 2008.

[8] RadioPulse LM2470/2475 RF4CE Technical Specification Datasheet, http://www.radiopulse.co.kr/, 2015.
[9] Microchip Application Notes, "AN1283: Microchip Wireless Media Access Controller MiMAC", "AN1284: Microchip Wireless Application Programming Interface MiApp" and "AN1066: Microchip Wireless Networking Protocol Stack MiMAC", http://www.microchip.com/miwi, 2011.

[10] T. Lennvall, S. Svensson and F. Hekland, "A comparison of WirelessHART and ZigBee for industrial applications", IEEE International workshop on Wireless Factory Communication Systems, pp. 85-88, 2008.

[11] Freescale Product Brief and development kits MC132X,http://www.element14.com/community/servlet/JiveServlet/ download/46712-2-98858/MC13242_RFFS.pdf,2010.

[12] C. Gomez, J. Oller and J. Paradells, "Overview and Evaluation of Bluetooth Low Energy: An Emerging Low-Power wireless technology.", Sensors Journal, vol. 12, no. 9, ISSN 1424-8220, pp.11734-11739, 2012.

[13] Nordic Semiconductor, "Multiprotocol Bluetooth low energy $/ 2.4 \mathrm{GHz}$ RF System on Chip - nRF51822 product specification", https://www.nordicsemi.com/.../nRF51822_PS v3.1.pdf

[14] 802.15.4, Part 15.4, "Wireless Medium Access Control (MAC) and Physical Layer (PHY) Specifications for Low-Rate Wireless Personal Area Networks (LRWPANs)".

[15] ZigBee Alliance, "ZigBeeRFCE specification: ZRC profile. Version 2.0 ZigBee", http://www.zigbee.org/zigbee-for-developers/networkspecifications/zigbeerf4ce/, 2014.

[16] P. Rohitha, P. R. Kumar, N. Adinarayana and V.N. Rao, "Wireless Networking Through ZigBee Technology", International Journal of Advanced Research in Computer Science and Soft. Eng. IJARCSSE, ISSN 2277-128X, vol. 2, no. 7, pp. 49-54, 2012.

[17] ZigBee Alliance, "ZigBee RFCE specification: ZigBee v.1.0", http://www.zigbee.org/zigbee-for-developers/networkspecifications/zigbeerf4ce/, 094945r00ZB 2010.

[18] WiMedia Specifications, "The WiMedia common Radio Platform", http://www.wimedia.org/en/docs/10001r01WM_MPI-

WiMedia_MAC-PHY Interface_Specification_1.5.pdf, Rel 1.5, 2009.

[19] E. Karapistoli, F.N. Pavlidou, I. Gragopoulos and I. Tsetsinas, "An overview of the IEEE 802.154a standard", IEEE Communications Magazine, ISSN 0163-6804, pp.47-52, Jan 2010.

[20] WG802.15 - Wireless Personal Area Network (WPAN) Working Group, "802.15.4-2011 - IEEE Standard for Local and metropolitan area networks-Part 15.4: Low-Rate Wireless Personal Area Networks (LR-WPANs)", IEEE standard, 2011.

[21] WG802.15- Wireless Personal Area Network (WPAN) Working Group, "802.15.4-2015 - IEEE Standard for Low-Rate Wireless Personal Area Networks (WPANs)", IEEE standard 2015.

[22] I. Howitt, J. A. Gutierrez, "IEEE 802.15.4 low rate - wireless personal area network coexistence issues", Wireless Communications and Networking, ISSN 1525-3511, vol. 3, pp. 1481-1486, 2003.

[23] HopeRF transceiver 433MHz RF12B datasheet, http://www.hoperf.com/upload/rf/RFM12B.pdf, 2012.

[24] HopeRF RF69W/RF69HW high range 433MHz transceiver datasheet, http://www.hoperf.com/upload/rf/RFM69CW-V1.1.pdf, 2015.

[25] MG-811 $\mathrm{CO}_{2}$ sensor datasheet, Sandbox Electronics, $\mathrm{http}: / /$ sandboxelectronics.com/?P=147,2011.

[26] Atmel ATmega328 8 bit microcontroller datasheet and user's manual, http://www.atmel.com/.../atmel-8271-8-bit-avr-microcontrolleratmega48a-48pa-88a-88pa-168a-168pa-328328p_datasheet_complete.pdf, 2015

[27] Atmel 128K/16KBytelow voltage operation EEPROM TWI chip, www.smartrobots.pl/download/AT24C256.pdf, 2012.

[28] AKBARI, Saba. Energy harvesting for wireless sensor networks review. In: Computer Science and Information Systems (FedCSIS), 2014 Federated Conference on. IEEE, 2014. p. 987-992. 